Arq. Bras. Med. Vet. Zootec., v.68, n.3, p.683-686, 2016

\title{
Microtia e atresia congênita do canal auditivo em cão: relato de caso
}

[Microtia and congenital external auditory canal atresia in dog: case report]

\author{
N.B. Messas, B.D.L. Cipriano, P.A.T. Andreussi, V.J.B. Terra; M.I.P. Palumbo*
}

Universidade Federal de Mato Grosso do Sul - Campo Grande, MS

\begin{abstract}
RESUMO
Descreve-se o caso de um cão, Pinscher, fêmea de 11 meses de idade, com alteração anatômica da orelha do lado direito. Após avaliação, foi realizado o diagnóstico clínico e o radiográfico de microtia com atresia do canal auditivo do lado direito. Como o animal não apresentava sinais de alterações no sistema vestibular ou otite, optou-se pelo acompanhamento clínico do caso. A microtia, caracterizada pela hipoplasia parcial ou completa da pina, assim como outras anomalias do conduto auditivo, é raramente descrita em medicina veterinária. De acordo com a revisão de literatura realizada, este é o primeiro relato ocorrido no Brasil de um cão com microtia e atresia congênita do canal auditivo sem a associação de síndrome vestibular.
\end{abstract}

Palavras-chave: cão, anomalia, alterações congênitas

\begin{abstract}
This paper describes an 11-month-old female Pinscher dog, with anatomical changes of the ear on the right side. After physical exam, clinical and radiographic diagnosis of microtia with ear canal atresia on the right side was made. As the animal did not show any signs of vestibular system changes or ear infections, the veterinarians opted for monitoring the patient. Microtia, characterized by the partial or complete hypoplasia of pina, as well as other abnormalities of ear canal are rarely described in veterinary medicine. As far as the author's knowledge by literature review carried out, this is the first report of a dog with microtia and congenital ear canal atresia not associated with vestibular syndrome in Brazil.
\end{abstract}

Keywords: dog, anomaly, congenital alterations

\section{INTRODUÇ̃̃̃O}

Anomalias congênitas do conduto auditivo são raramente diagnosticadas na medicina veterinária (Simpson, 1997; Coomer e Bacon, 2009). A microtia é caracterizada por hipoplasia de pina, que varia de aplasia parcial à completa. Perda da audição e atresia auricular congênita são anomalias comumente encontradas com microtia. Rezaei et al. (2014) relataram um caso de microtia congênita com atresia do canal auditivo em um cão com dois meses de idade e Simpson (1997) relatou um caso de atresia do meato acústico externo relacionada à dor otológica. A ocorrência de alterações nos nervos vestibulococlear e facial também foram observadas em um cão com atresia unilateral do

Recebido em 23 de julho de 2015

Aceito em 15 de fevereiro de 2016

*Autor para correspondência (corresponding author)

E-mail: mariana.palumbo@ufms.br conduto auditivo e inflamação crônica supurativa secundária ao acúmulo de cerúmen (Béraud, 2012).

A etiologia dessas anomalias congênitas e seu desenvolvimento ainda não são esclarecidos (Santos et al., 2009; Luquetti et al., 2012; Rezaei et al., 2014). A atresia de canal auditivo pode ser originada de forma congênita ou traumática (Coomer e Bacon, 2009), porém, quando diagnosticada em animais jovens, provavelmente é de origem congênita (Caine et al., 2008).

O diagnóstico baseia-se no exame físico minucioso de recém-nascidos e realização cuidadosa de otoscopia em animais aparentemente saudáveis. A radiografia evidencia estenose do canal auditivo e aumento 
da opacidade da bula timpânica (Rezaei et al., 2014). Já a tomografia computadorizada revela que a parte vertical do canal auditivo termina de maneira abrupta (Schmidt et al., 2007).

O tratamento da atresia do conduto auditivo é cirúrgico, baseado na reconstrução do conduto (Simpson, 1997). A escolha da realização do procedimento cirúrgico deve ser fundamentada em diversos fatores, entre eles a localização da atresia, o grau de alterações no conduto auditivo externo, a presença ou a ausência de otite média e sinais neurológicos e da cronicidade das lesões (Béraud, 2012). A atresia do canal auditivo favorece a instalação de processo inflamatório devido ao acúmulo de restos celulares e cerume. Tendo em vista que as anomalias congênitas do conduto auditivo são raras e considerando a falta de descrição dessas alterações em medicina veterinária, o objetivo deste trabalho é relatar um caso de microtia congênita do canal auditivo em cão, não associada à síndrome vestibular.

\section{CASUÍSTICA}

Foi atendido no Hospital Veterinário da Universidade Federal de Mato Grosso do Sul (UFMS) da Faculdade de Medicina Veterinária e Zootecnia (FAMEZ) um animal da espécie canina, Pinscher, 11 meses de idade, fêmea. A queixa clínica principal era oftálmica, diagnosticada como blefarite, porém, durante o exame físico, foi observada alteração anatômica do pavilhão auricular direito, caracterizada por hipoplasia de pina direita com estenose do conduto auditivo e cartilagens da orelha externa subdesenvolvidas (Fig. 1A). Não foram observadas alterações anatômicas na orelha esquerda (Fig. 1B) nem alterações nos parâmetros físicos, incluindo-se o exame neurológico. Da mesma forma, o hemograma encontrava-se dentro dos limites de normalidade. Não havia sinais clínicos compatíveis com otite média ou interna, como meneio cefálico, secreção auricular, prurido e otalgia. A estenose do conduto auditivo impossibilitou exame mais detalhado. Foi realizado exame radiográfico do crânio para avaliação das bulas timpânicas e condutos auditivos, que revelou diminuição e opacificação da bula timpânica direita quando comparada com a esquerda (Fig. 1C e 1D), além de estenose do conduto auditivo externo direito (Fig. 1E).

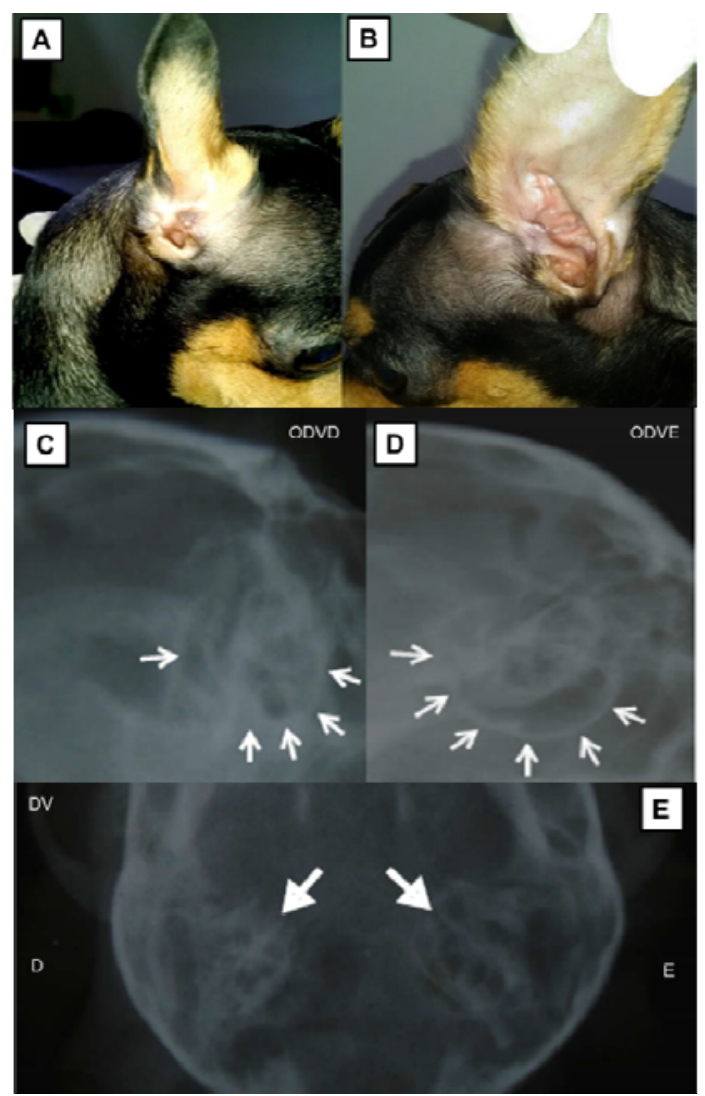

Figura 1. A) Pavilhão auditivo direito com alterações anatômicas. Observa-se hipoplasia de pina e diminuição da abertura externa do conduto auditivo. B) Pavilhão auricular esquerdo sem alterações anatômicas. C) Projeção radiográfica oblíqua dorsoventral esquerda da bula timpânica. Observa-se diminuição e opacificação da bula timpânica do lado direito (setas) quando comparada ao lado esquerdo (figura D). D) Projeção radiográfica oblíqua dorsoventral direita da bula timpânica. Observa-se tamanho e opacificação normais da bula timpânica esquerda (setas). E) Projeção radiográfica dorsoventral do crânio. Diminuição e opacificação na bula timpânica direita, quando comparada com a esquerda (setas).

\section{DISCUSSÃO}

As alterações anatômicas do sistema auditivo são raramente descritas em animais (Béraud, 2012; Palumbo et al., 2013; Rezaei et al., 2014). Apesar do cão deste relato não apresentar nenhuma comorbidade, sabe-se que as alterações na conformação da orelha podem estar 
associadas a lesões no sistema vestibular (Béraud, 2012; Palumbo et al., 2013), a episódios de otalgia (Simpson, 1997) e à inflamação crônica supurativa da orelha média e interna (Béraud, 2012). Nos relatos de Simpson (1997) e Rezaei et al. (2014), os cães não apresentavam sinais neurológicos, corroborando os achados clínicos deste relato, já que o animal foi atendido com queixa de prurido periocular, sem sinais compatíveis com lesões no sistema vestibular, otite média ou trauma. O primeiro relato de microtia em cão realizado no Brasil foi em um canino sem raça definida, com 23 dias de idade, que também apresentava síndrome vestibular periférica (Palumbo et al., 2013).

No caso descrito, foi diagnosticada a presença de microtia e atresia de conduto auditivo direito, de acordo com um estudo realizado em humanos por Santos et al. (2009) e Wu et al. (2010), que afirmam maior ocorrência de microtia unilateral do lado direito. $\mathrm{Na}$ medicina veterinária, as alterações anatômicas do sistema auditivo relatadas em cães por Simpson (1997), House (2001), Schmidt et al. (2007), Coomer e Bacon (2009), Béraud (2012), Palumbo et al. (2013) e Rezaei et al. (2014) também eram do lado direito, e apenas Caine et al. (2008) relataram alterações anatômicas no conduto auditivo esquerdo. De acordo com os artigos revisados, ainda não se sabe a razão da ocorrência ser maior do lado direito, uma vez que a etiologia permanece desconhecida (Rezaei et al., 2014). Além disso, provavelmente isto não tenha nenhuma implicação clínica para o animal.

Os achados radiográficos deste trabalho também foram observados em outros casos relatados de microtia e atrofia do conduto auditivo. O exame radiográfico realizado por Rezaei et al. (2014) demonstrou estenose do canal auditivo direito, e a vista lateral do crânio revelou leve espessamento e aumento da opacidade da bula timpânica do lado com microtia. No caso relatado por Simpson (1997), as radiografias do crânio demonstraram ausência de ar na área normalmente ocupada pelo conduto auditivo externo direito e o canal não podia ser distinguido dos tecidos moles adjacentes devido ao aumento da opacidade da bula timpânica, bem como a sua parede óssea, que estava espessada e esclerótica. Em um caso de atresia do conduto auditivo externo relatado por Caine et al. (2008) em um Red Setter, radiograficamente o canal auditivo externo esquerdo não estava preenchido por ar e a bula timpânica apresentou radiopacidade de tecido mole.

O tratamento deve ser feito com reconstrução cirúrgica da orelha (Schmidt et al. 2007; Caine et al. 2008; Santos et al. 2009). Schmidt et al. (2007) relataram resultado satisfatório após cirurgia reconstrutiva de um caso de atresia do meato acústico externo em um Labrador Retriever. A reconstrução cirúrgica do canal auditivo também já foi relatada em um gato com atresia congênita do canal auditivo externo de três anos de idade (Coomer e Bacon, 2009). Neste caso, como a alteração não estava afetando a qualidade de vida do animal, a proprietária optou por não realizar intervenção cirúrgica no momento do diagnóstico.

Alguns autores acreditam que as taxas de incidência de alterações congênitas nas orelhas, especialmente a microtia, são subestimadas devido à pouca atenção dedicada ao exame físico realizado em recém-nascidos e à não realização de otoscopia em animais atendidos com outras queixas clínicas (Béraud, 2012; Rezaei et al., 2014). Dessa maneira, apesar de os casos de atresia do canal auditivo serem raros, é fundamental a avaliação detalhada do sistema auditivo com auxílio de otoscópios na rotina de atendimento aos animais. O cão deste relato foi atendido por alteração oftálmica e o diagnóstico de microtia e atresia do conduto auditivo só foi feito devido ao exame físico detalhado realizado durante a consulta. De acordo com a revisão de literatura, este é o primeiro relato realizado no Brasil de um cão com microtia e atresia congênita do canal auditivo sem a associação de síndrome vestibular ou outros sinais relacionados.

\section{CONCLUSÃO}

Este trabalho ressalta a importância da avaliação física neonatal durante o atendimento clínico a cães e gatos, pois, muitas vezes, os animais com alterações anatômicas congênitas são atendidos com queixas clínicas não relacionadas à malformação. A realização da otoscopia e o conhecimento das possíveis deformidades relacionadas ao sistema auditivo são fundamentais para diagnóstico precoce das anomalias congênitas otológicas de cães. 


\section{REFERÊNCIAS}

BÉRAUD, R. Surgical management of 2 different presentations of ear canal atresia in dogs. Can. Vet. J., v.53, p.412-418, 2012.

CAINE, A.R.; HERRTAGE. M.E.; LADLOW, J.F. Imaging diagnosis: ear canal distension following external auditory canal atresia. Vet. Radiol. Ultrasound, v.49, p.267-269, 2008.

COOMER, A.; BACON, N. Primary anastomosis of segmental external auditory canal atresia in a cat. J. Feline Med. Surg., v.11, p.864 $-868,2009$.

HOUSE, A. Atresia of the distal external acoustic meatus in a Bouvier des flandres. $J$. Small Anim. Pract., v.42, p.88-89, 2001.

LUQUETTI, D.V.; HEIKE, C.L.; HING, A.V. et al. Microtia: epidemiology and genetics. Am. J. Med. Genet. Part A, v.158, p.124-139, 2012.

PALUMBO, M.I.P.; CONTI, J.P.; LOURENÇO, M.L.G.; MACHADO, L.H.A. Síndrome vestibular periférica congênita associada à microtia em cão. Vet. Zootec., v.20, p.270-274, 2013.
REZAEI, M.; MAHMOUDI, T.; EBRAHIMI, M.; VOSUGH, D. First report of microtia in dog. Comp. Clin. Pathol., v.24, p.699-702, 2015.

SANTOS, G.B.; VASCONCELLOS, J.J.A.; VIEIRA, V.J. et al. Perfil epidemiológico dos pacientes com microtia do serviço de cirurgia plástica HU-UFSC. Arq. Catarinenses Med., v.38, p.27-29, 2009.

SCHMIDT, K.; PIAIA, T.; BERTOLINE, G.J.; DE LORENZI, D. External auditory canal atresia of probable congenital origin in a dog. J. Small Anim. Pract., v.48, p.233-6, 2007.

SIMPSON, D. Atresia of the external acoustic meatus in a dog. Aust. Vet. J. v.75, p.18-20, 1997.

WU. J.; ZHANG, R.; ZHANG, Q. et al. Epidemiological analysis of microtia: a retrospective study in 345 patients in China. Int. J. Pediatric Otorhi., v.74, p.275-278, 2010. 